\title{
EMPHYSEMATOUS PYELONEPHRITIS - CLINICORENTGENOLOGIC DIAGNOSIS, REQUIRING URGENT SURGICAL TREATMENT CASE REPORT
}

\author{
Dyakov Sv. $^{1}$, A. Hinev ${ }^{1}$, M. Siderova ${ }^{2}$, H. Bohchelian ${ }^{2}$, K. Hristozov ${ }^{2}$, V. Platikanov ${ }^{3}$ \\ ${ }^{1}$ UMHAT "St. Marina” Varna, Third Clinic of Surgery, Division of Urology, ${ }^{2}$ UMHAT "St. \\ Marina" Varna, Clinic of Endocrinology and Metabolic Diseases, ${ }^{3}$ UMHAT "St. Marina" Varna, \\ Clinic of Anaesthesiology and Intensive Care
}

Reviewed by: Assoc. Prof. K. Nenov, MD, PhD

\begin{abstract}
Emphysematous pyelonephritis is a rare and life-threatening suppurative infection of the renal parenchyma and the perirenal tissues, characterized by spontaneous gas production. Although uncommon, it occurs almost exclusively in diabetic patients $(60-80 \%$ of the cases). We describe a recent case of a diabetic woman with emphysematous pyelonephritis, managed by unilateral nephrectomy. While the symptoms are usually general and nonspecific, the diagnostic approach is crucial in many cases. Ultrasonography should be the first diagnostic tool, as it is noninvasive, fast, and cost effective. However, CT scan is more specific and sensitive, and it should always be taken into consideration, as it enables the proper treatment decisions.
\end{abstract}

Keywords: emphysematous pyelonephritis, diabetes mellitus, ketoacidosis, ultrasound

\section{INTRODUCTION}

Emphysematous pyelonephritis (EPN) is a rare, rapidly progressive, necrotizing infection, which is mainly characterized by gas production in the renal parenchyma and the perirenal tissues. It occurs almost exclusively in diabetic patients, which constitute $60-80 \%$ of the cases $[2,5]$. However, $15 \%$ of the patients present without a prior history of diabetes $[1,6]$. Other risk factors, associated with EPN, include: diabetic ketoacidosis, polycystic kidneys, renal failure, cirrhosis, alcoholism, and malnutrition. Mortality is within the range between 25 to $80 \%$, and it is even higher in case of bilateral kidney involvement.

We describe a recent case of a diabetic woman with emphysematous pyelonephritis, managed by unilateral nephrectomy.

\section{CASE PRESENTATION}

A 63-year-old female (PIR, Medical Record No 30554 / 6.11.2007) with 20-year history of diabetes mellitus and hypertonic disease was admitted to our hospital. She com-

Address for correspondence:

Svetoslav Dyakov. Department of Surgery, Division of Urology, Varna Medical University, 55 Marin Drinov Str., Varna 9002, Bulgaria.

E-mail: sve_dyakov@yahoo.com plained of fever, chills, nausea, chest and abdominal pain for the past few days. At admission the patient was conscious but confused, febrile up to $39^{\circ} \mathrm{C}$, tachydyspnoic, tachycardic, hypotonic with blood pressure $85 / 40 \mathrm{mmHg}$. The abdomen was tympanic on percussion, with slow peristalsis, no guarding, rigidity or rebound tenderness, the left side was painful on palpation. The left flank was painful on percussion. There were some depressions of the ST-segment on ECG. Therefore, the patient was first admitted in the ICU ward. A few hours later she was transferred to the Clinic of Endocrinology, because no myocardial infarction was present and high levels of blood glucose were detected in the serum. Her laboratory results were as follows: Hemoglobin $112 \mathrm{~g} / \mathrm{l}$, WBC $19.000 / \mathrm{mm}^{3}, 202000$ platelets $/ \mathrm{mm}^{3}$, high cholesterol and triglycerides, BUN 22, Creatinine $231 \mathrm{mcmol} / 1$, serum blood glucose $25 \mathrm{mmol} / \mathrm{l}$. Blood gas analysis showed metabolic acidosis. The urine was positive for glucose and protein, and the sediment was rich in WBC. Urine and blood cultures were negative (however, they both were performed upon triple antibiotic treatment regimen). In the next 36-48 hours the patient became comatose, her general common condition gradually worsened, despite the antibiotic and insulin therapy. Consultations with neurologist and pulmologist were performed. Following the consultation of a nephrologist and the US of the abdomen, a consultation with urologist was done on day 3 .

Diagnostic imaging

Brain CT: It was performed after a consultation with neurologist in order to identify any organic cause for the comatose state of the patient. The brain CT showed no abnormalities. 
Ultrasound of the abdomen: It showed gas collection in the left kidney area, and in the urinary bladder.

CT of the abdomen: On the whole body scan the stomach and the bowels were full of gas, and in the area of the upper pole of the left kidney a gas collection was visible/Fig. 1/. Transversal scans showed gas in the parenchyma and subcapsular gas collection in the upper-dorsal area of the left kidney /Fig. 2/.

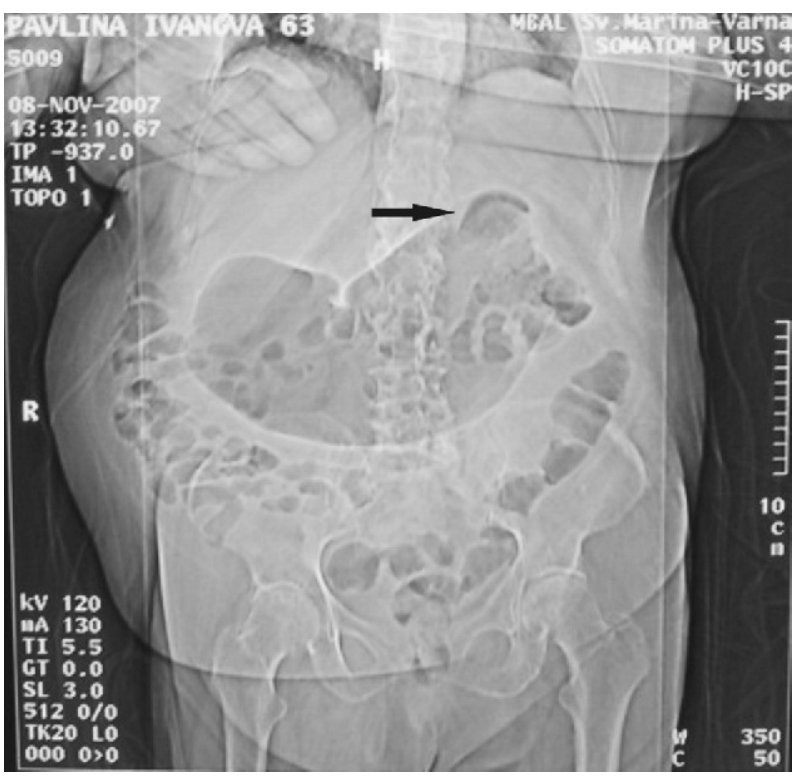

Fig. 1. Whole body scan, showing gas collection in the stomach and the bowels, as well as in the area of the upper pole of the left kidney (arrow).

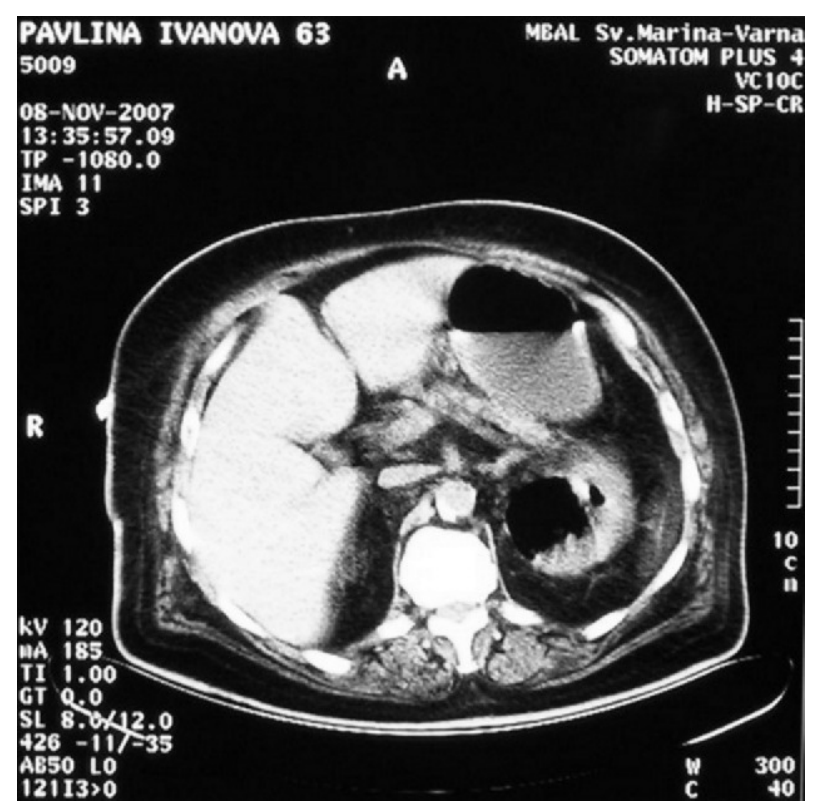

Fig. 2. Transversal CT scan, showing gas collection in the left kidney.

\section{Diagnosis}

The clinical signs were nonspecific, showing evidence of infection as the main cause for the uncontrolled blood glucose levels. US as a noninvasive, cheap and easy to ap- proach diagnostic procedure led us to the initial diagnosis. The final diagnosis of emphysematous pyelonephritis was made when the abdominal CT was performed. The patient was consulted by an urologist, who took a decision for immediate surgical treatment. Because of the developing septic shock, an urgent nephrectomy was performed.

\section{Intraoperative findings}

The left kidney was approached via transversal laparotomy, cutting the posterior peritoneal layer. The kidney and the perirenal tissues were infiltrated and inflamed. The ureter and the renal vein and artery were ligated and cut. At the time of the extraction of the kidney, the renal capsule at the upper pole (at the site of the gas collection) was torn. A sudden, loud crackle was heard and gas with unpleasant odor spread in the operation theatre. The section of the specimen revealed small amount of pus in the renal pelvis. The boundaries of the gas cavity were evident in the upper-rear subcapsular region, compressing the renal parenchyma /Fig. 3/.

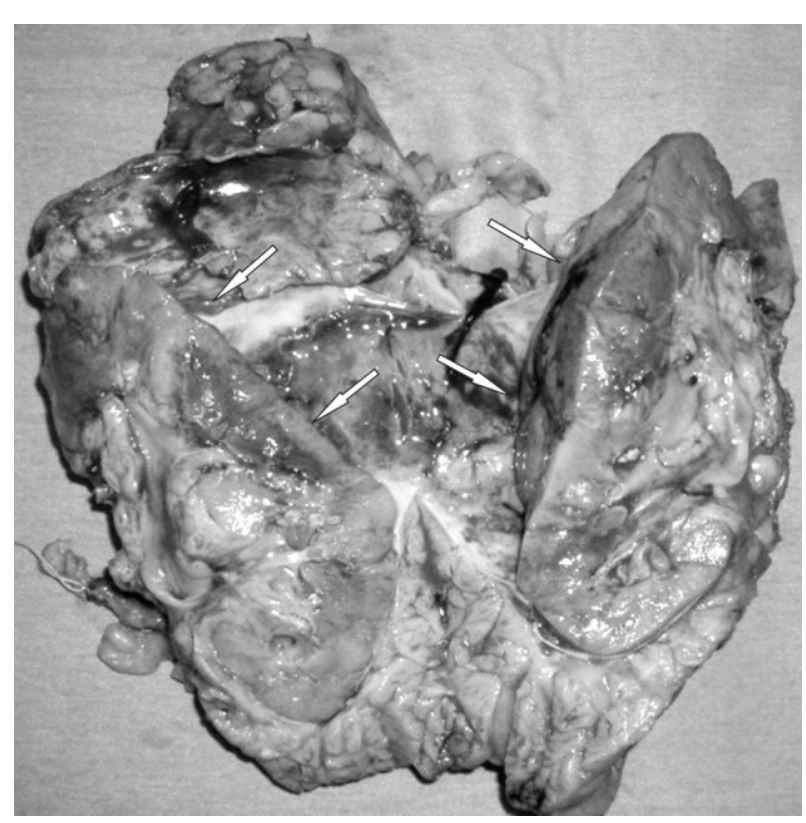

Fig. 3. Cross section of the surgical specimen. The boundaries of the gas cavity are shown by arrows.

\section{Hystological findings}

Histological specimen (№ 12506-10 / 14.11.2007) showed evidence of chronic inflammatory process with necrosis that invaded the perirenal and pararenal tissues.

\section{Postoperative period}

After surgery, the patient was admitted in the Clinic of Anesthesiology and Intensive Treatment. The operative wound healed primarily. The drainage tubes and the sutures were removed in the usual terms. Despite the lack of surgical problems, the stabilizing of the febrile state and the normalized levels of blood glucose, the patient remained comatose and unable to regain spontaneous breathing. Several attempts to be extubated and left to breathe spontaneously were done. However, after any such attempt, just a few hours later, she had to be intubated again, because of 
the low saturation. Tracheotomy had to be performed, due to the long intermittent positive pressure ventilation (IPPV). Despite all these intensive cares, the respiratory distress syndrome (RDS) could not be overcome, and the patient died on the $50^{\text {th }}$ day after surgery.

\section{DISCUSSION}

The first case of emphysematous pyelonephritis was published over 100 years ago [3]. Since then, sporadic cases of EPN were reported in the literature [1-6].

E. coli (69\%), Klebsiella pneumonia (29\%) and Proteus are the most commonly microorganisms isolated from the blood, or from the urine [6]. The presence of gas is attributed to rapid glucose fermentation by gas producing bacteria. Previous studies have shown that the most common gas is nitrogen, followed by oxygen, hydrogen, and carbon dioxide $[1,2,4,6]$

Diagnosis of EPN can be made by plain X-ray, sonography, although CT scan is more specific and sensitive [4]. As our case illustrates, sonogram gave us the initial diagnosis and was the reason for a CT. Due to the prevalence of diabetic patients among those with EPN, and the high mortality rate of EPN $[2,5]$ we recommend US of the abdomen to be performed immediately after admission. US is noninvasive, relatively cheap and easy-to-approach diagnostic tool. When EPN is suspected on US, a CT should be made to confirm the diagnosis. Unfortunately, the US in our case was made 48 hours after the patient was brought to the hospital.

Radiologically, four classes of emphysematous pyelonephritis are described on computed tomography [2]. In EPN Class 1 and 2, the gas is localized in the collecting renal system and in the renal parenchyma, respectively, without extension to the extrarenal space. In EPN Class 3A, as seen in this case, there is extension of gas into the perinephric space, and in Class 3B, to the pararenal space. Bilateral EPN, or EPN of a solitary kidney, represent the most severe forms of the disease (Class 4).

Emphysematous pyelonephritis is associated with a high mortality rate $(40 \%)$ when treated with antibiotics alone [2]. Although milder forms of the disease (Class 1 and 2) have been successfully treated by a combination of percutaneous renal drainage and antibiotics, these modalities alone may be insufficient in more severe presentations of the disease, or in patients presenting with septic shock. In such patients, early nephrectomy is recommended as the method of choice [1,2].

The patient in our case had emphysematous pyelonephritis that clinically resembled ketoacidosis with abdominal pain. The patient was febrile and appeared toxic. Her abdomen was diffusely tender with dilated stomach and colon, tympanic on percussion and decreased bowel sounds. US was suspicious for emphysematous pyelonephritis with gas detected in the kidney and in the bladder. The CT scan proved to be essential in making the definitive diagnosis.

\section{CONCLUSION}

Emphysematous pyelonephritis is an uncommon, life-threatening entity, which should always be suspected in a febrile toxic diabetic patient. The combined use of immediate diagnostic US with CT or MRI, followed by prompt surgical intervention, could be lifesaving.

\section{REFERENCES}

1. Abdul-Halim, H., E. Kehinde, S. Abdeen, I. Lashin, A. Al-Hunayaa, K. Al-Awadi. Severe emphysematous pyelonephritis in diabetic patients. Urol Int. 2005, 75(2), 123-128.

2. Huang J., C. Tseng. Emphysematous pyelonephritis: clinicoradiological classification, management, prognosis, and pathogenesis.Arch Inter Med. 2000, 160(6), 797-805.

3. Kelly, H., W. Mac Callum. Pneumaturia. JAMA. 1898, 31, 375.

4. Kuo, Y., M. Chen, G. Liu, C. Huang, C. Huang, C. Huang. Emphysematous pyelonephritis: imaging diagnosis and follow-up. Kaohsiung J Med Sci. 1999, 15(3), 159-170.

5. McHugh, T., S. Albanna, N. Stewart. Bilateral emphysematous pyelonephritis. Am J Emerg Med. 1998, 16(2), 166-169.

6. Muttarak, M., W. Mai. Clinics in diagnostic imaging. Left emphysematous pyelonephritis. Singapore Med J. 2004, 45(7), 340-342. 